\title{
POLARIMETRIC CALIBRATION OF THE SPACEBORNE DIRECTIONAL POLARIMETRIC CAMERA INSTALLED ON THE GF-5 SATELLITE
}

\author{
Yuan Yinlin ${ }^{1}$, Liu Zhen ${ }^{2,3},{ }^{*}$, Zheng Xiaobing ${ }^{1}$, Kang Qing ${ }^{1}$, Wu Haoyu ${ }^{1}$, Li Jianjun ${ }^{1}$, Li Shuang ${ }^{1}$, Luo Donggen ${ }^{1}$, \\ Hong Jin ${ }^{1}$
}

\begin{abstract}
${ }^{1}$ Key Laboratory of Optical Calibration and Characterization, Anhui Institute of Optics and Fine Mechanics, Chinese Academy of Sciences, Hefei, Anhui 230031, China - (ylyuan, xbzheng, qkang, hywu, jjli, shli, dgluo, hongjin)@aiofm.ac.cn ${ }^{2}$ Institute of Artificial Intelligence and Big Data, Hefei University, Hefei, Anhui 230601, China - liuzhen@hfuu.edu.cn ${ }^{3}$ Combinatorial Optimization and Graph Algorithms, Institute of Mathematics, Technische Universität Berlin, Berlin 10623, Germany - liu@math.tu-berlin.de
\end{abstract}

KEY WORDS: Polarimetric calibration, polarization sensitivity, non-uniform response, calibration accuracy

\begin{abstract}
:
The Spaceborne Directional Polarimetric Camera (SDPC) is dedicated to obtain an accurate information of clouds and microphysical properties of aerosol particles via multi-angle, multi-spectral and multi-polarization observations. It images the Earth with $110^{\circ}$ field of view (FOV) in 3 polarimetric spectral bands and 5 non-polarimetric spectral bands from visible to near infrared. Due to its wide FOV telecentric optical system and multi-channels, polarization sensitivity of optical components (PSOC) and non-uniform response of pixels (NURP) are the main uncertainty factors of polarimetric calibration. In this paper, a polarimetric calibration model of SDPC has been constructed for increasing the measurement accuracy. Combined an integrating sphere with a polarization generator as source, PSOC can be measured in high accuracy by using the Fourier series analysis method which reduces the impact form the alignment error of the generator. The sectional viewing field measurement method is used to acquire NURP while the large aperture integrating sphere served as reference source. Subsequently, the data of NURP have been corrected by the relative transmittance of high frequency and low frequency respectively for polarized channels. The result of validation experiment shows that the polarization measurement errors in $0^{\circ}, 15^{\circ}, 30^{\circ}$ and $45^{\circ}$ half field of view (HFOV) are all less than $0.5 \%$ when the degree of linear polarization of reference source is larger than 0.1 .
\end{abstract}

\section{INTRODUCTION}

SDPC (Spaceborne Directional Polarimetric Camera) (Huang et al., 2014; Luo et al., 2018; Qian, 2017; Qian et al., 2017; Yang et al., 2015) is a polarimeter designed for accurately acquiring clouds and aerosol particle microphysical properties via multi-angle, multi-spectral and multi-polarization observations. SDPC is developed by Anhui Institute of Optics and Fine Mechanics which installed on the GF-5 satellite and launched in May 2018 (Kang, 2018; Kang et al., 2019; Yuan et al., 2019). Both along-track and cross-track instantaneous viewing field of SDPC are $110^{\circ}$. It has an ultra-wide field of view (FOV) telecentric lenses, a rotating wheel bearing filter and a two-dimensional CCD $512 \times 512$ pixels detector array. The continuously rotating wheel, installed between optical components and CCD detector, discriminates 15 measurement channels. 1 channel is dedicated to the detector dark current response measurement; the other 14 channels are fitted with spectral filters and 9 of them with added polarizers.

In order to obtain an accurate polarization of aerosol particles (Duan et al., 2007; Duan et al., 2008; Duan et al., 2017; Frank et al., 2002; Leroy et al., 1997), the accuracy of degree of linear polarization (DolP) measurement of SDPC should be better than $0.5 \%$ (Bretdibat et al.,1995; Chenault et al., 1999; Durieux et al., 1994; Fougnie et al., 2007). When light transmitted through the ultra-wide FOV telecentric optical system, its polarization state altered (Chen ,2008; Chen et al., 2010; Chen et al.,2011; Chen, 2015; Tang et al.,2008; Sun et al., 2005) owing to the polarization sensitivity of optical components (PSOC). Likewise, the non-uniform response pixels (NURP) result in variations of relative transmittance of high frequency and low frequency due to the manufacturing flaw of optical components. Obviously, PSOC and NURP should be considered two important factors in the polarimetric calibration of SDPC.

In this paper, a polarimetric calibration model for polarized channels of SDPC has been constructed. Subsequently, a geometric calibration system and the Fourier series analysis method are used to obtain PSOC. In addition, the sectional viewing field measurement method is applied to acquire the relative transmittance of high frequency and low frequency for calibration of NURP. Finally, a polarimetric calibration accuracy experiment is designed for validation and verification of this model.

\section{POLARIMETRIC CALIBRATION MODEL}

The optical system of SDPC is shown in Figure 1. It is composed of three parts: an ultra-wide-angle image telecentric optical lenses, a rotating wheel bearing filter and a $512 \times 512$ pixels CCD detector array. The ultrawide FOV telecentric imaging optical lens consists of 20 optical lenses with multiple optical surfaces. The rotating wheel bearing filters has 15 channels. 1 channel for measuring the dark current. Spectral bands of 5 unpolarized observation channels are $443 \mathrm{~nm}, 550 \mathrm{~nm}$, $763 \mathrm{~nm}, 765 \mathrm{~nm}, 910 \mathrm{~nm}$. On the other hand, spectral bands of 3 polarized observation are $490 \mathrm{~nm}, 670 \mathrm{~nm}$ and $865 \mathrm{~nm}$, and each of them has 3 channels respectively 
equipped with $0^{\circ}, 60^{\circ}$ and $120^{\circ}$ polarizers. Through rotating the wheel bearing, polarization information of the target is obtained.

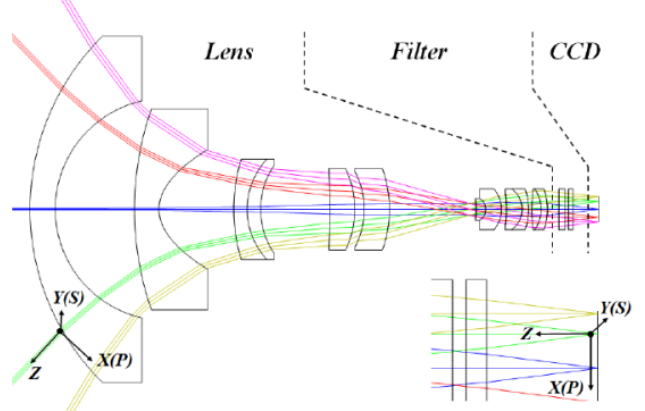

Figure 1. Layout of the SDPC optical structure

Due to the oblique incidence, the ultra-wide-angle telecentric optical lenses produce polarization sensitivity. The polarization sensitivity changes the polarization states of incident beams which significantly impact the polarimetric calibration accuracy. Previous experimental results (Che et al., 2009; Li et al., 2007; Sun et al., 2010; Wang et al., 2014) indicate that the maximum transmission direction of ultra-wide-angle telecentric optical lenses is in the meridional plane, which formed by chief ray and optical axis. The chief ray is defined as the light that passes through the center of the aperture stop from the off-axis point. Correspondingly, the minimum transmission direction is in the sagittal plane, which is perpendicular to the maximum transmission direction. As shown in Fig. 2, the maximum transmission direction is determined by the projection of meridional plane on the plane where polarizers located.

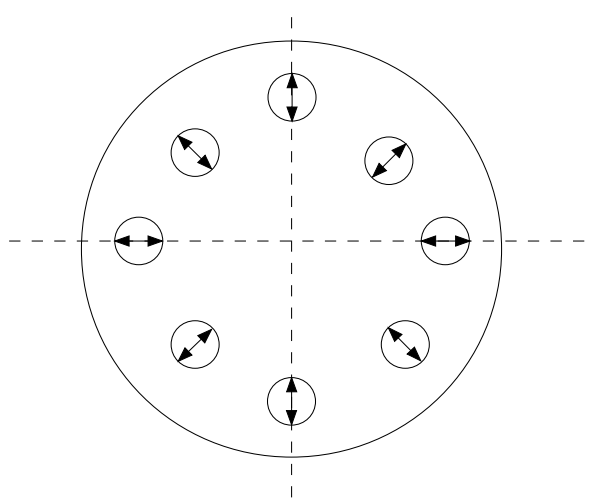

Figure.2. The maximum and minimum transmission directions of ultra-wide-angle telecentric optical lenses

By combined the maximum and minimum transmission directions as the reference coordinate system, a Mueller matrix is constructed for the description of polarization sensitivity of optical components. Removed polarizers from the optical structure, define the Stokes vector as $S$, ${ }_{l, p}^{k, a}=\left[\begin{array}{lll}I_{l, p}^{k, a} & Q_{l, p}^{k, a} & U_{l, p}^{k, a}\end{array}\right]$ (There is no V component in the vector because the SDPC does not detect the circular polarization). It reaches to the CCD detector array can be expressed as

$$
\begin{gathered}
S_{l, p}^{k, a}=\left[\begin{array}{c}
I_{l, p}^{k, a} \\
Q_{l, p}^{k, a} \\
U_{l, p}^{k, a}
\end{array}\right]=\left[\begin{array}{ccc}
1 & \varepsilon_{l, p}^{k, a} & 0 \\
\varepsilon_{l, p}^{k, a} & 1 & 0 \\
0 & 0 & J_{\varepsilon}
\end{array}\right]\left[\begin{array}{c}
I_{l, p}^{k, a} \\
Q_{l, p}^{\prime k, a} \\
U_{l, p}^{\prime k, a}
\end{array}\right] \\
J_{\varepsilon}=2 \frac{\sqrt{\left(1-\varepsilon_{l, p}^{k, a}\right) /\left(1+\varepsilon_{l, p}^{k, a}\right)}}{1+\left(1-\varepsilon_{l, p}^{k, a}\right) /\left(1+\varepsilon_{l, p}^{k, a}\right)}
\end{gathered}
$$

As shown in Eqs. (1) and (2), where

$k=$ spectral band

$a=$ channel number $(a=1,2,3, a \in \mathrm{N})$

$l, p=$ two-dimensional coordinative position of the pixel

$S_{l, p}^{k, a}=$ Stokes vector reaches to the CCD detector array with no polarizer in the optical structure $I_{l, p}^{k, a}=$ measured intensity of the pixel which coordinates position is $(l, p)$

$Q_{l, p}^{k, a}=$ amount of linear horizontal or vertical polarization.

$U_{l, p}^{k, a}=$ amount of linear $+45^{\circ}$ or $-45^{\circ}$ polarization.

$I_{l, p}^{k, a}, Q_{l, p}^{k, a}, U_{l, p}^{k, a}=$ components of Stokes vector

which represent polarization states of object lights

$\varepsilon_{l, p}^{k, a}=$ polarization sensitivity of optical components (PSOC)

The relationship between the measured intensity $I_{l, p}^{k, a}$ and the incident intensity $I_{l, p}^{k, a}$ is

$$
I_{l, p}^{k, a}=\frac{\gamma}{2} I_{l, p}^{{ }^{k, a}}\left(1+\varepsilon_{l, p}^{k, a} \cos 2 \chi\right)
$$

where $\gamma$ denotes maximum transmission. $\chi$ denotes the angle between the vibration direction of incident light and the direction of the maximum transmission of optical lenses. It can be seen from Eq. (3) that the ultra-wideangle telecentric optical lenses obviously change the polarization state of the oblique incident light. The polarimetric calibration model for polarized channels of SDPC can be described as

$$
\left[\begin{array}{c}
I_{l, p}^{k, a} \\
Q_{l, p}^{k, a} \\
U_{l, p}^{k, a}
\end{array}\right]=\tilde{M}_{l, p}^{k-1}\left[\begin{array}{c}
\left(D N_{l, p}^{k, 1}-D C_{l, p}^{k}\right) \\
\left(D N_{l, p}^{k, 2}-D C_{l, p}^{k}\right) \\
\left(D N_{l, p}^{k, 3}-D C_{l, p}^{k}\right)
\end{array}\right]
$$

where $D N_{l, p}^{k, a}=$ response of polarized channel $a$ in spectral band $k$

$D C_{l, p}^{k}=$ dark current of spectral band $k$

$M_{l, p}^{k}=$ Measurement matrix, defined as

$\tilde{M}_{l, p}^{k}=\frac{1}{A^{k} \cdot t^{s} \cdot d_{l, p}^{k}}\left[\begin{array}{lll}T^{k, 1} \cdot g_{l, p}^{k, 1} \cdot P_{1}^{k, 1}(l, p) & T^{k, 1} \cdot g_{l, p}^{k, 1} \cdot P_{2}^{k, 1}(l, p) & T^{k, 1} \cdot g_{l, p}^{k, 1} \cdot P_{3}^{k, 1}(l, p) \\ T^{k, 2} \cdot g_{l, p}^{k, 2} \cdot P_{1}^{k, 2}(l, p) & T^{k, 2} \cdot g_{l, p}^{k, 2} \cdot P_{2}^{k, 2}(l, p) & T^{k, 2} \cdot g_{l, p}^{k, 2} \cdot P_{3}^{k, 2}(l, p) \\ T^{k, 3} \cdot g_{l, p}^{k, 3} \cdot P_{1}^{k, 3}(l, p) & T^{k, 3} \cdot g_{l, p}^{k, 3} \cdot P_{2}^{k, 3}(l, p) & T^{k, 3} \cdot g_{l, p}^{k, 3} \cdot P_{3}^{k, 3}(l, p)\end{array}\right]$

where $\quad A^{k}=$ the absolute calibration coefficient of the spectral band $k$, which is measured by an integrating sphere light source with large aperture 
$t^{s}=$ exposure time, and $s$ represents observation mode (ocean mode, land mode and userdefined observation mode)

$T^{k, a}=$ relative transmittance

$d_{l, p}^{k}=$ relative transmittance of low frequency for each pixel

$\mathrm{g}_{\mathrm{l}, \mathrm{p}}^{\mathrm{k}, \mathrm{a}}=$ relative transmittance of high frequency for each pixel

$P_{1}^{k, a}(l, p), P_{2}^{k, a}(l, p)$ and $P_{3}^{k, a}(l, p)=$ polarization response function of polarizers, described as

$$
\left\{\begin{array}{c}
P_{1}^{k, 1}(l, p)=1+\eta^{k} \varepsilon_{l, p}^{k, a} \cos 2\left(\varphi_{l, p}-\alpha^{k, a}\right) \\
P_{2}^{k, 1}(l, p)=\eta^{k} \cos 2\left(\varphi_{l, p}-\alpha^{k, a}\right)+\varepsilon_{l, p}^{k, a} \\
P_{3}^{k, 1}(l, p)=\eta^{k} \sin 2\left(\varphi_{l, p}-\alpha^{k, a}\right)
\end{array}\right.
$$

where $\quad \eta^{k}=$ extinction ratio of polarizers

$\varphi_{l, p}=$ azimuth which is corresponding to two-

dimensional coordinative position $(l, p)$ on

CCD detector array

$\alpha^{k, a}=$ actual polarizer azimuth

\section{PSOC MEASUREMENT}

The optical system of SDPC is not an ideal linear system. Ultra-wide-angle image telecentric optical lenses produce distortion, and distortion sizes of various spectral bands are different. However, the same mathematical geometry model can be used for scaling, except that coefficients in different geometric models vary with wavelength (Wu and Song, 2007; Zou et al., 2006)

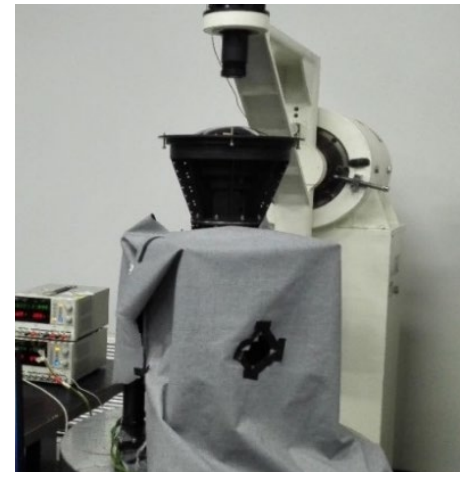

As shown in Fig. 3, point $\mathrm{O}$ indicates the center of the CCD detector array. $\mathrm{S}_{\mathrm{k}}$ indicates the center of distortion corresponding to the k-band. $\theta$ indicates the FOV, and $\varphi$ indicates the angle between the projection of the FOV and the $\mathrm{X}$ axis.

The center of distortion can be written as

$$
\left\{\begin{array}{c}
X M=X S_{K}-\cos \varphi^{*} S_{K} M \\
Y M=Y S_{K}-\sin \varphi^{*} S_{K} M \\
S_{K} \vec{M}=f 1_{k} \operatorname{tg} \theta+f 3_{k} \operatorname{tg}^{3} \theta+f 5_{k} \operatorname{tg}^{5} \theta
\end{array}\right.
$$

where $\quad\left(X S_{k}, Y S_{k}\right)=$ the coordinate of the distortion center

$f 1_{k}, f 3_{k}, f 5_{k}=$ distortion indexes, respectively Apparently, the PSOC $\varepsilon_{l, p}^{k, a}$ at the position of the coordinate point $(l, p)$ on the CCD detector array is only related to the FOV $\theta$.

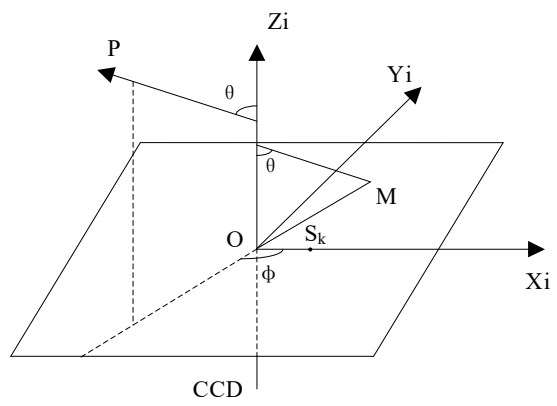

Figure 3. Wide FOV geometric calibration model

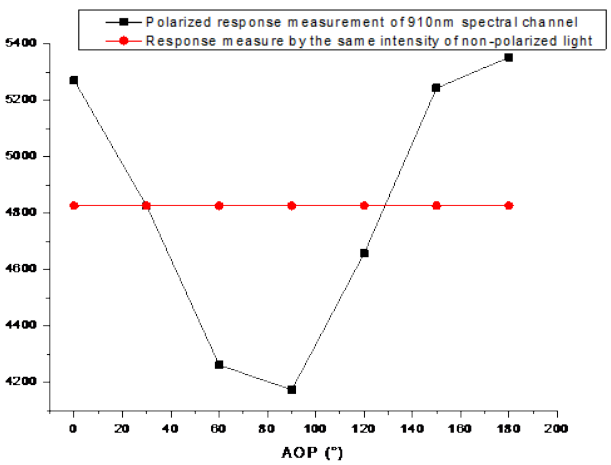

Figure 4. The geometric calibration system for PSOC measurement (left) and PSOC measurement values in $56^{\circ} \mathrm{FOV}$ of 910nm channel (right)

As shown in Fig.4 (left), the geometric calibration system is constituted of geometric calibration platform and polarized source. It provides collimated parallel beams with known FOV $\theta$, DolP and AolP (azimuth of liner polarization). By using the geometric calibration system, coordinates of these parallel beams on the CCD detector array is derived, and the relationship between the PSOC and five FOV calibration parameters $X S_{k}, Y S_{k}$, $f 1_{k}, f 3_{k}$ and $f 5_{k}$ are measured simultaneously. The polarized source includes an integrating sphere source and a rotating polarizer. In order to measure the PSOC, we use the polarized source to produce linear polarized light with invariable intensity and DolP but AolP continuously varying from $0^{\circ}$ to $180^{\circ}$ at $30^{\circ}$ intervals. the measurement results of PSOC in different AOLs are shown in Fig.4 (right). The shape of the curve indicates that PSOC and AolP approximately satisfy the harmonic function relationship. Consider that the orientation error of reference polarized source may influence measurement results, the Fourier series analysis method is used to calculate the PSOC in a certain FOV, that is

$$
\varepsilon_{\theta}^{k}=\frac{2}{P} \frac{\sum_{i=0}^{N}\left(D N_{\theta}^{k}-D C_{\theta}^{k}\right) \cos 2 \frac{i \pi}{N}}{\sum_{i=0}^{N}\left(D N_{\theta}^{k}-D C_{\theta}^{k}\right)}
$$

where $\quad k=$ spectral channel 
$\theta=$ field of view (FOV)

$\varepsilon_{\theta}^{k}=$ PSOC measured in a certain FOV

$P=$ degree of linear polarization $(\operatorname{Dol} P)$

$D N_{\theta}^{k}=$ average polarized response (DN) in $\theta$ viewing field

$D C_{\theta}^{k}=$ dark current response $(\mathrm{DN})$

According to measured values in 18 FOVs, the PSOC is obtained by fitting the 7 th power polynomial. As shown in Eq. (9), the fit of PSOC in all fields of view is

$$
\varepsilon_{\theta}^{k}=A+\sum_{i=1}^{7} B_{i} \times \theta^{i}
$$

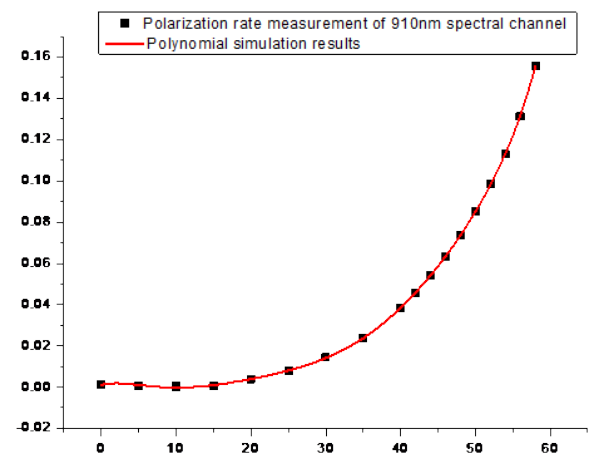

Measured values of PSOC in 18 different FOVs and polynomial simulation to establish PSOC in the full FOV are shown in Figure 5 (left) and Figure 5 (right), respectively. The simulation result shows that the max value of PSOC is 0.176 (the vertical scale is 10000:1). It means that when the SDPC measured by the fully linearly polarized light, the radiometric calibration uncertainty caused by the PSOC is about $8.8 \%$. For high precision radiometric observation applications, it usually required that the radiometric calibration uncertainty is less than 5\%. Based on Eq. (1) and simulation result of PSOC, the radiometric calibration uncertainty is decreased from $8.8 \%$ to 2.2\% (Qian et al., 2017).

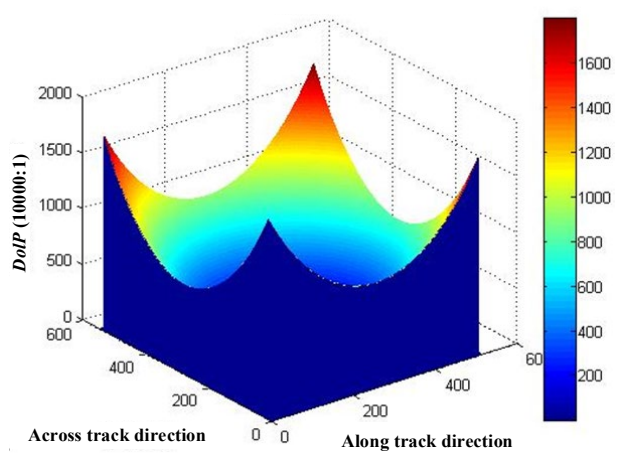

Figure 5. DolP measurement in 18 viewing fields (left) and DolP measurement model (right)in full FOV

\section{NURP MEASUREMENT}

As shown in Fig. 6, The NURP measurement system comprises an integrating sphere with large aperture (Yuan et al., 2013; Yuan, 2014) and a spectral radiometer. We choose the sectional viewing field measurement

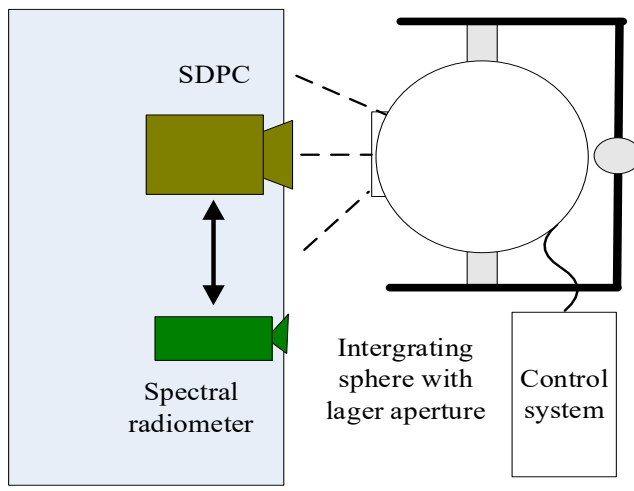

method to detect the NURP because (a) the FOV inconsistency among 3 polarized channels caused by the wedge-shaped lenses could be avoided. (b) NURP stemmed from different incident angles of the integrating sphere source would be removed.

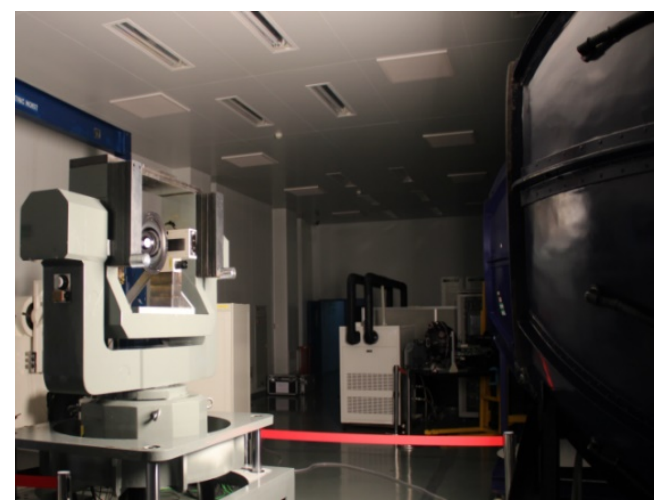

Figure 6. The schematics of NURP measurement system(left) and the real object of it (right)

Fig. 7 shows measurement results of NURP in SDPC's 490nm P3 channel at test position matrix $7 \times 7$ (left) and $15 \times 15$ (right). Obviously, NURP produced measurement error. In the Fig. 7 (left), the relative deviation of response in the sectional splice gap is about $0.5 \%$. In the Fig. 7 (right), there is no sectional splice gap, but the bone image is clearly distinguishable, which is inherent feature of the CCD detector. The maximum difference between bone image and fringe areas is about $0.7 \%$. In order to correct the NURP, we analyze it in the relative transmittance of low frequency and the relative transmittance of high frequency. 

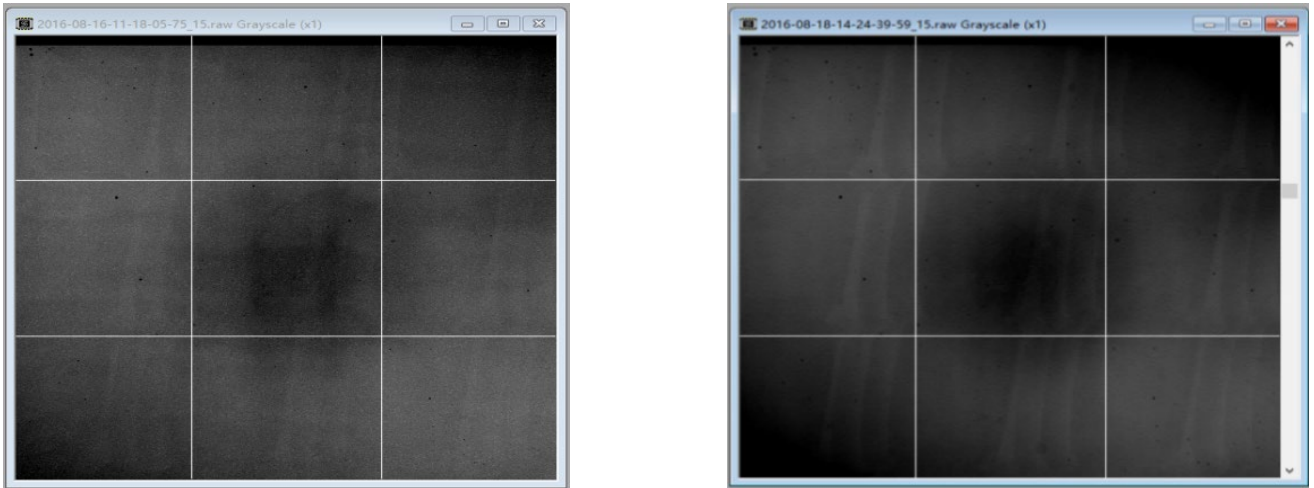

Figure 7. NURP measurement of SDPC 490nm P3 channel with test position matrix $7 \times 7$ (left) and $15 \times 15$ (right)

The relative transmission $T^{k, a}$ is described as

$$
T^{k, a}=\frac{\sum_{l=l_{0}-1}^{l_{0}+1} \sum_{p=p_{0}-1}^{p_{0}+1}\left(D N_{l, p}^{k, a}-D C_{l, p}^{k}\right)}{\sum_{l=l_{0}-1}^{l_{0}+1} \sum_{p=p_{0}-1}^{p_{0}+1}\left(D N_{l, p}^{k, 2}-D C_{l, p}^{k}\right)}
$$

The relative transmittance of low frequency $d_{l, p}^{k}$ represent non polarized light transmission of sectional viewing field. For polarized channels, the interval angles of three analyzers are $0^{\circ} 、 60^{\circ}$ and $120^{\circ}$, respectively. According to orthogonal characteristic of trigonometric function, $d_{l, p}^{k}$ can be described as

$$
d_{l, p}^{k}=\frac{\sum_{l=l_{0-1}}^{l_{s}+1} \sum_{p=p_{\rho-1}}^{p_{e}+1} \operatorname{mean}\left(X_{l, p}^{k, 1}+X_{l, p}^{k, 2}+X_{l, p}^{k, 3}\right)}{3 * \sum_{l=l_{0}-1}^{l_{0}+1} \sum_{p=p_{0}-1}^{p_{0}+1} \operatorname{mean}\left(X_{l, p}^{k, 2}\right)}
$$

where $X_{l, p}^{k, a}(a=1,2,3)$ is data processing response of $k$ spectral band, via
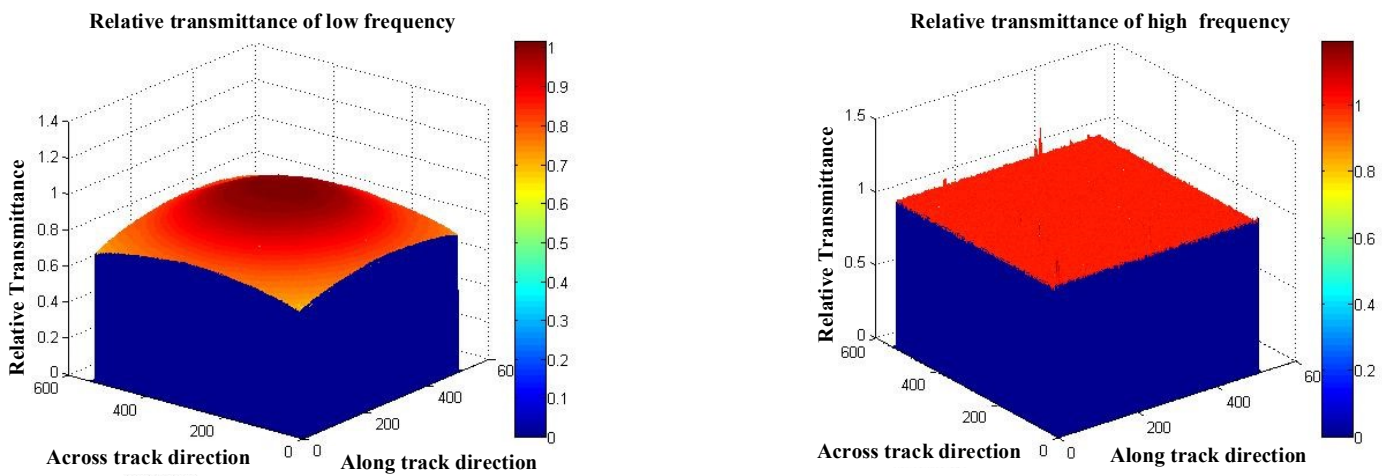

Figure 8. Measurement results of the relative transmittance of low frequency (left) and relative transmittance of high frequency in DPC 865nm P1 polarized channel (right)

\section{VALIDATION EXPERIMENT AND ANALYSIS}

In order to test the validity of the SDPC polarimetric calibration model, the VPOLS (high-precision variable polarization light source) (Chen et al., 2012; Kang, 2015) is used to carry out the validation experiment. VPOLS is

$$
X_{l, p}^{k, a}=\left(D N_{l, p}^{k, a}-D C_{l, p}^{k}\right) / T^{k, a}
$$

Using the data processing response $X_{l, p}^{t, a}$, the relative transmittance of high frequency $g_{l, p}^{k, a}$ can be calculated via

$$
g_{l, p}^{k, a}=\frac{3 * X_{l, p}^{k, a}}{\sum_{l=l_{\rho}}^{l_{p}+1} \sum_{p=p_{\rho}}^{p_{p}+1} \operatorname{mean}\left(X_{l, p}^{k, 1}+X_{l, p}^{k, 2}+X_{l, p}^{k, 3}\right)}
$$

The relative transmittance of low frequency $d_{l, p}^{k}$ and the relative transmittance of high frequency $g_{l, p}^{k, a}$ have been measured in $865 \mathrm{~nm}$ P1 polarized channel, and their measurement results are shown in Fig. 8. The unit of row and column coordinates represent the distribution of pixels on CCD detector array, and the vertical coordinate indicates the relative transmittance. Protrusion and descent in the graph are mainly caused by the difference between overheated pixels and normal pixels. 
polarization state adjuster includes a three-degree-offreedom rotating electromechanical driving mechanism and high precision polarizing glass piles, as shown in Fig. 9. By adjusting the output wavelength and polarization states, VPOLS produces linear polarization rays through four parallel glass plates, and the Dolp adjustment range varies from $0 \%$ to $72 \%$ within spectral band from $0.46 \mu \mathrm{m}$ to $2 \mu \mathrm{m}$.

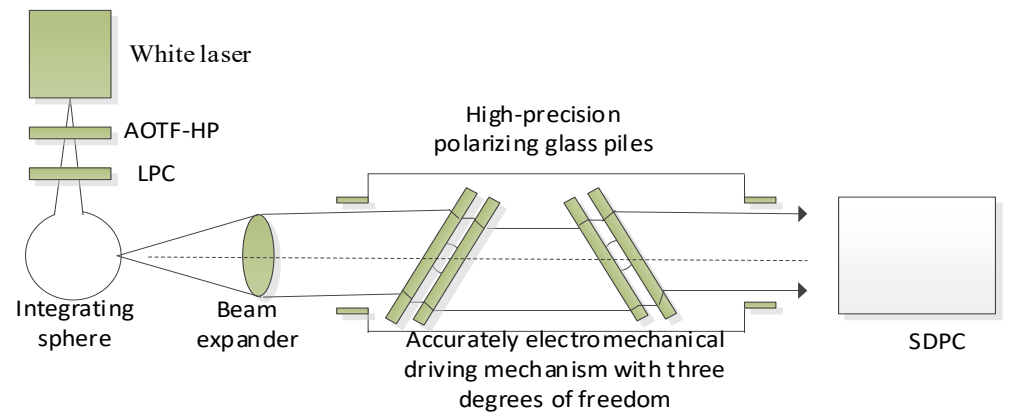

Figure 9. The layout of the VPOLS

The absolute deviation $\Delta P^{k}$ between numerical calculated values of VPOLS and measurement values of the SDPC is calculated via

$$
\Delta P^{k}=\left|P_{M}^{k}(\theta)-P_{C}^{k}\right|
$$

Fig. 10 shows the experiment that uses VPOLS to check the measurement accuracy of SDPC. As can be seen from Table 1, when the output DolP of VPOLS varies from $10 \%$ to $40 \%$, the absolute deviation $\Delta P^{k}$ of $0^{\circ}, 15^{\circ}, 30^{\circ}$ and $45^{\circ}$

HFOV in $670 \mathrm{~nm}$ band are measured. The max deviations are $0.13 \%, 0.44 \%, 0.29 \%$, and $0.33 \%$, respectively. Therefore, the polarization calibration accuracy of SDPC is less than $0.5 \%$. However, when the output DolP of the VPOLS is $0 \%$, that is, natural light, the deviation of each FOV in $670 \mathrm{~nm}$ band is larger than $1 \%$. The reason is that when four sheets of glass are all in a vertical position, the VPOLS outputs natural light, and the light is reflected back and forth between the SDPC lenses and the flat glass, which introduces errors in the

\begin{tabular}{|c|c|c|c|c|c|c|c|c|}
\hline $\mathrm{HFOV}\left({ }^{\circ}\right)$ & \multicolumn{2}{|c|}{$0^{\circ}$} & \multicolumn{2}{|c|}{$15^{\circ}$} & \multicolumn{2}{|c|}{$30^{\circ}$} & \multicolumn{2}{|c|}{$45^{\circ}$} \\
\hline $\mathrm{P}_{\mathrm{C}}^{670 \mathrm{~nm}}(\%)$ & $\mathrm{P}_{670 \mathrm{~nm}}(\%)$ & $\begin{array}{c}\Delta \mathrm{P}^{670 \mathrm{~nm}} \\
(\%)\end{array}$ & $\mathrm{P}_{M}(\%)$ & $\begin{array}{c}\Delta \mathrm{P}^{670 \mathrm{~nm}} \\
(\%)\end{array}$ & Рм(\%) & $\begin{array}{c}\Delta \mathrm{P}^{670 \mathrm{~nm}} \\
(\%)\end{array}$ & $\mathrm{P}_{M}(\%)$ & $\begin{array}{c}\Delta \mathrm{P}^{670 \mathrm{~nm}} \\
(\%)\end{array}$ \\
\hline 0.00 & 1.25 & 1.25 & 1.08 & 1.08 & 1.08 & 1.08 & 1.66 & 1.66 \\
\hline 10.00 & 10.13 & 0.13 & 10.44 & 0.44 & 10.29 & 0.29 & 10.33 & 0.33 \\
\hline 15.00 & 15.04 & 0.04 & 15.09 & 0.09 & 15.17 & 0.17 & 15.16 & 0.15 \\
\hline 20.00 & 20.10 & 0.10 & 19.95 & 0.05 & 20.18 & 0.18 & 20.14 & 0.14 \\
\hline 25.00 & 25.08 & 0.08 & 24.94 & 0.06 & 25.17 & 0.17 & 25.12 & 0.12 \\
\hline 30.00 & 30.11 & 0.11 & 29.98 & 0.02 & 30.23 & 0.23 & 30.09 & 0.09 \\
\hline 40.00 & 40.13 & 0.13 & 40.14 & 0.14 & 40.29 & 0.29 & 40.07 & 0.07 \\
\hline $\begin{array}{c}\operatorname{Max} \Delta \mathrm{P}^{670 \mathrm{~nm}} \\
(10.00-40.00)\end{array}$ & \multicolumn{2}{|c|}{0.13} & \multicolumn{2}{|c|}{0.44} & \multicolumn{2}{|c|}{0.29} & \multicolumn{2}{|c|}{0.33} \\
\hline
\end{tabular}

Table 1 Test results of DolP in 670nm

In order to solve this problem, it is necessary to use LEAIS (large exit aperture integrating spheres reference light source) to measure the polarization calibration accuracy of the full FOV. First, we use spectral polarization analyzer (Yuan et al., 2014) to check the polarization of the LEAIS. The DolP produced by the LEAIS $865 \mathrm{~nm}$ band in the full FOV is shown in Fig. 11 process of measurement.

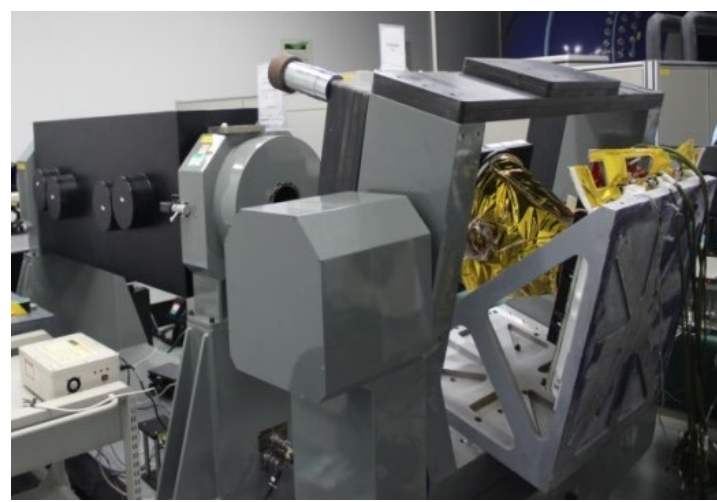

Figure 10. Polarimetric calibration accuracy validation experiment with VPOLS 
contamination on the polarizer or filter combination wheel. However, points spreading around edges whose

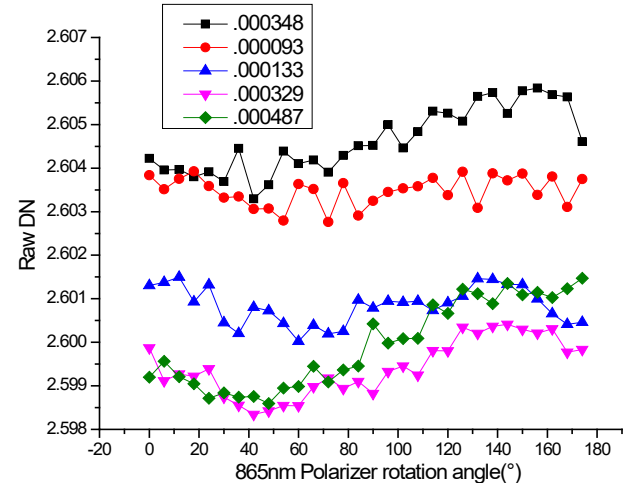

values are larger than $0.5 \%$ caused by unknown origin, which needs further research.

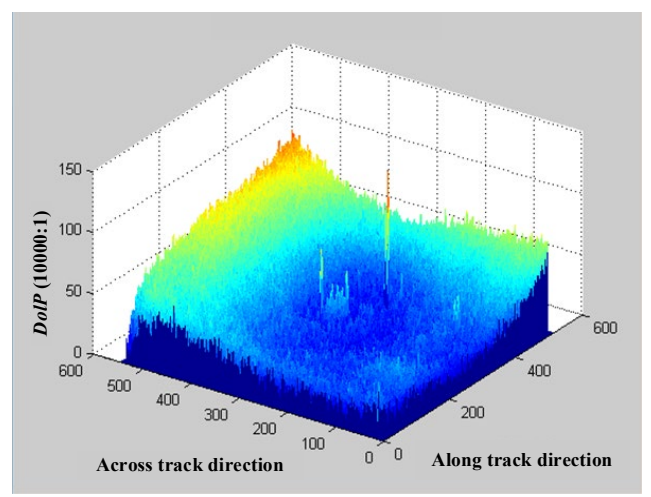

Figure 11. The polarization produced by LEAIS $865 \mathrm{~nm}$ in the full FOV (left) and polarimetric calibration accuracy validation results of SDPC $865 \mathrm{~nm}$ channel tested by LEAIS (right).

\section{CONCLUSION}

In this work, a new polarimetric calibration model of SDPC has been proposed for analyzing PSOC and NURP. By using a geometric calibration system, PSOC of 18 FOVs has been measured. A fitting of 7 th power polynomial is obtained to simulate the distribution of the PSOC of full FOV. The simulation result plays a key role in decreasing radiometric calibration uncertainty from $8.8 \%$ to $2.2 \%$. The NURP is corrected by the relative transmission of high and low frequency, which are measured by sectional viewing field measurement method. The validation experiment result shows that polarimetric calibration accuracy in $0^{\circ}, 15^{\circ}, 30^{\circ}$ and $45^{\circ}$ HFOVs is better than $0.5 \%$ while DolP of reference light source is varied from $10 \%$ to $40 \%$. Whereas, when the DolP of reference light is less than $0.05 \%$, under the condition of full FOV test, some points at the center and edge of CCD detector array are larger than $0.5 \%$, and the reason will be considered in further research. We preliminaries validate this polarimetric calibration method is applicable for meeting the high-precision calibration requirements for scientific application.

\section{ACKNOWLEDGEMENTS}

The authors would like to acknowledge the support of the National Natural Science Foundation of China under Grants No.41605021, the Talent Research Fund Project of Hefei University (16-17RC20), and the National Key R\&D Program of China (2018YFB0504602).

\section{REFERENCES}

Bretdibat, T., Andre, Y., Laherrere, J.M., 1995. Preflight calibration of the POLDER instrument. Proc. SPIE 2553, 218-231. doi.org/10.1117/12.221357.

Cremer, F., Jong, W., Schutte, K., 2002. Infrared polarization measurements and modeling applied to surface-laid antipersonnel landmines. Opt. Eng. 41, 1021-1032. doi.org/ 10.1117/1.1467362.

Chen, L.G., 2008: Study of Laboratory Calibration of the airborne polarization CCD Camera with Wide Field of View. Anhui Institute of Optics and Fine Mechanics.
Che, H.Z., Zhang, X.Y., Chen, H.B., Damiri, B., Goloud, P., Li, Z.Q., Zhang, X.C., Wei, Y., Zhou, H.G., Dong, F., Li, D.P., Zhou, T.M., 2009. Instrument calibration and aerosol optical depth validation of the China Aerosol Remote Sensing Network. J. Geophys. Res.-Atmos. 114, 1-12. doi.org/10.1029/2008JD011030.

Chen, L.G., Meng, F.G.,Yuan, Y.L., Zheng, X.B., 2010. Project of calibration method for polarization camera. Journal of atmospheric and environmental optics, 5(3), 227 231. doi.org/10.3969/j.issn.1673-6141.2010.03.009.

Chen, L.G., Meng, F.G.,Yuan, Y.L., Zheng, X.B., 2011. Experimental study for the polarization characteristics of airborne polarization camera. Journal of Optoelectronics Laser, 22(11), 1629 1632. doi.org/1005-0086(2011)111629-04.

Chen, L.G., Meng, F.G., Yuan, Y.L., Zheng, X.B., 2012. High-precision variable polarization light source. Procedia Engineering 29, 1835-1839. doi.org/10.1016/j.proeng.2012.01.222.

Chen, L.G., 2015. Polarimetric Calibration of the Polarization CCD Camera with Large Viewing Field.

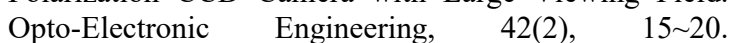
doi.org/10.3969/j.issn.1003-501X.2015.02.003.

Durieux, A., Neubert, S., Petitbon I., 1994. POLDER: a wide field-of-view instrument for earth-polarized observation. Proc. SPIE 2209, 160-169. doi.org/10.1117/12.185253.

Duan, M.Z., Lv, D.R., 2007. Simultaneously Retrieving Aerosol Optical Depth and Surface Albedo over Land from POLDER's Multi-angle Polarized Measurements I: Theory and Simulations. Chinese Journal of Atmospheric Sciences, 31(5), 757-765. doi.org/ CNKI:SUN:DQXK.0.2007-05-000.

Duan, M.Z., Lv, D.R., 2008. Simultaneously Retrieving Aerosol Optical Depth and Surface Albedo over Land from POLDER's Multi-angle Polarized Measurement. II: A Case Study. Chinese Journal of Atmospheric Sciences, 32(1), 27-35. doi.org/ 10.3878/j.issn.10069895.2008.01.03. 
Fougnie, B., Bracco, G., Lafrance, B., Ruffel, C., Hagolle, O., Tinel, C., 2007. PARASOL in-flight calibration and performance. Appl. Optics 46, 54355451. doi.org/10.1364/AO.46.005435.

Huang, H.L., Yi, W.N., Qiao, Y.L., 2014. Validation of retrieving aerosol optical parameters over the sea using airborne directional polarized camera. Acta Optica Sinica, 34(6), 0601004. doi.org/ 10.3788/AOS201434.0601004.

Kang, Q., 2015: Design and Test of Variable Polarization Light Source with Large Dynamic Range. Anhui University.

Kang, Q., 2018: Research on System-Level Radiometric and Polarized Calibration Methods in Laboratory of Polarization Remote Sensors. University of Science and Technology of China.

Kang, Q., Yuan, Y.L., Weng, J.W., Ding, L., Li, J.J., Wu, H.Y., Hong, J., Zheng, X.B., 2019. System-Level Polarized Calibration Methods in Laboratory of Directional Polarization Camera. Journal of atmospheric and environmental optics, 14(1), 36 48. doi.org/ $10.3969 /$ j.issn.1673-6141.2019.01.004.

Leroy, M., Deuzé, J. L., Bréon, F. M., Hautecoeur, O., Herman, M., Buriez, J. C., Tanré, D., Bouffiès, S., Chazette, P., Roujean, J. L., 1997. Retrieval of atmospheric properties and surface bidirectional reflectances over land from POLDER/ADEOS. J. Geophys. Res. 102, 17023-17037. doi.org/ 10.1029/96JD02662.

Labonnote, L. C., Brogniez, G., Gayet, J.F., Buriez, J.C., Boucher, M. D., 1999. Modeling of polarized light scattering in cirrus clouds: validation with in-situ measurements and ADEOS-POLDER reflectance observations. Proc. SPIE 3754. doi, org/10.1117/12.366341.

Li, Z.Q., Goloub, P., Blarel, L., Damiri, B., Podvin, T., Jankowiak, I., 2007. Dust optical properties retrieved from ground-based polarimetric measurements. Appl. Optics 46, 1548-1553. doi.org/10.1364/AO.46.001548.

Luo, D.G., 2017: Design and Research of Photoelectric Detection System for Directional Polarization Camera. University of Science and Technology of China.

Qian, H.H., 2017: Laboratory full field of view polarization calibration of directional polarimetric camera. University of Science and Technology of China.

Qian, H.H., Meng, B.H., Yuan, Y.L., Hong, J., Zhang, M.M., Li, S., Qiu, Z.W., 2017. Full field of view polarization effect measurement and error analysis of non-polarized channels of Spaceborne Directional Polarimetric Camera. Acta Phys. Sin. ,66 (10), 1007011-00701-10. doi.org/ 10.7498/aps.66.100701.

Sun, X.B., Hong, J., Qiao, Y.L., Yang, W.F., Luo, R.Z., 2005. nalysis of characteristic of multiband aerial polarization image. SPIE, 5832, 219 227. doi.org/ $10.1117 / 12.619661$

Sun, X.B., Qiao, Y.L., Hong, J., 2010. Review of Polarization Remote Sensing Techniques and Applications in the Visible and Infrared. Journal of atmospheric and environmental optics, 5(3), 175-189. doi.org/ 10.3969/j.issn.1673-6141.2010.03.002.

Tang, W.P., Hong, J.,Wang, Y.J., Yang, W.F., 2008. Airborne directional polarization camera and its optical system design. Journal of atmospheric and environmental optics, 3(3), 213 216. doi.org/ 1673-6141(2008)030212-05.

Wu, G.D., Song, D., 2007. Calibration of mapping camera and cubic prism coordinate system. Optics and Precision Engineering, 15(11), 1727-1730. doi.org/ 10.3321/j.issn:1004-924x.2007.11.015.

Wang, H., Sun, X.B., Sun, B., Hong, J., 2014. Evaluation of Land Surface Polarization Models Based on Airborne Advanced Atmosphere Multi-Angle Polarization Radiometer Measurement. Acta Optica Sinica, 34(1), 44251. doi.org/ 10.3788/AOS201434.0128002.

Yuan, Y.L., Xu, J., Zhai, W.C., Qi, T., Zou, P., Wu, H.Y., Zheng, X.B., 2013. Design and Test of a Spectrumtunable Integrating Spheres Reference Light Source with Large Exit Aperture. Acta Optica Sinica, 33(7), 0712004-1 0712004-8.

doi.org/10.3788/AOS201333.0712004.

Yuan, Y.L., 2014: Design and Application of a Spectrally tunable Integrating Sphere Reference Source with Large Exit Aperture. University of Chinese Academy of Sciences.

Yuan, Y.L., Zheng, X.B., Wu, H.Y., Meng, F.G., Kang, Q., Chen, L.G., Li, J.J., Zou, P., 2014. An intensity modulated spectro-polarimetric analyzer based on Fourier transformation. Acta Optica Sinica , 34(12), 1223001-1 1223001-7.

doi.org/10.3788/AOS201434.1223001.

Yang, W.F., Hong, J., Qiao, Y.L., 2015. Optical design of spaceborne directional polarization camera. Optical Design of Spaceborne Directional Polarization Camera. Acta Optica Sinica. 35(8), 0822005-1 04120038.doi.org/ 10.3788/AOS201535.0822005.

Yuan, Y.L., Kang, Q., Weng, J.W., Ding, L., Li, J.J., Li, S., Wu, H.Y., Hong, J., Zheng, X.B., 2019. Highprecision Radiometric Calibration of Directional Polarization Camera. Journal of atmospheric and environmental optics, 14(1), 24 35. doi.org/ 10.3969/j.issn.1673-6141.2019.01.003.

Zou, Y.P., Xu, S.P., Li, Y.F., Lin, Y.C., 2006. Comparison of laboratory testing of aerial camera interior orientation elements anddistortion calculation method. Geomatics Technology and Equipment. 8, 37-38. doi.org/CNKI:SUN:CHKJ.0.2006-01-012. 